\title{
LA FORMACIÓN DE ANIMADORES Y LÍDERES JUVENILES EN INSTITUCIONES EDUCATIVAS DE MONTEVIDEO
}

\author{
TRAINING OF YOUTH ANIMATORS AND LEADERS IN EDUCATIONAL INSTITUTIONS IN \\ MONTEVIDEO
}

Ricardo Lema Álvarez ${ }^{1}$

Resumen

El presente artículo es un avance de la investigación sobre la recreación educativa como trayecto de formación, que da cuenta de un conjunto de prácticas de recreación organizada en instituciones de educación formal en Uruguay. En este contexto, la formación de líderes y animadores juveniles constituye una de las propuestas emblemáticas de la oferta recreativa de estas instituciones. Este fue un estudio cualitativo, desde un enfoque de teoría fundamentada que supone el desarrollo de teoría a partir de los datos que surgen de la realidad estudiada. Con base en entrevistas a diez referentes de instituciones educativas que cuentan con estas propuestas, el artículo presenta algunos hallazgos sobre la forma de organización de estas propuestas, del impacto formativo en los adolescentes que participan en ellas y de la función pedagógica que se asume a partir de este rol voluntario.

Palabras claves: recreación educativa; formación de animadores; tiempo libre escolar

\section{Abstract}

This is a preliminary report of a research on recreation as an educational path, which shows organized recreation practices in some formal educational institutions in Uruguay. In this context, the training of youth leaders and animators represents one of the emblematic proposals of the recreational offer these institutions have. This was a qualitative study, based on a grounded theory approach that involves the development of theory from the data that emerged from the reality studied. Based on ten interviews conducted to school authorities that have these offers, the article presents some findings on how to organize those proposals, the formative impact on adolescents who participate on them, and the educational role that is assumed from this volunteer role.

Keywords: educational recreation; training of animators; school free time

Fecha de recepción: 20 de octubre de 2015

Fecha de aprobación: 8 de marzo de 2016

Para citar este artículo:

Lema, R. (2016). La formación de animadores y líderes juveniles en instituciones educativas de Monte-video. Lúdica Pedagógica, (23), 53-62.

1 Candidato a doctor en Ocio y Desarrollo Humano (Universidad de Deusto, España), licenciado en Comunicación Social con posgrado de Educación en Valores (Universidad Católica del Uruguay). Es docente e investigador del Departamento de Educación, Universidad Católica del Uruguay. Correo electrónico: rlema@ucu.edu.uy 


\section{INTRODUCCIÓN}

Hablar de recreación en el caso de Uruguay remite a un conjunto de prácticas vinculadas al juego y la expresión, que movilizan a diversos grupos y colectivos, en diferentes ámbitos, edades y clases sociales. Pero también la recreación remite a una dimensión de acción organizada, que desde diversas instituciones educativas, comunitarias y socioculturales, moviliza a grupos de todas las edades en torno a juegos, fiestas, campamentos y otras actividades que tienen en el horizonte la vivencia de experiencias satisfactorias.

La recreación organizada, especialmente desde un enfoque educativo, tiene un desarrollo destacado en Uruguay. La presencia de la recreación en las instituciones educativas de este país, a partir de una amplia oferta que acompaña las diferentes etapas del ciclo escolar pero por fuera del horario académico, constituye una experiencia que entendemos particular. En este contexto, la formación de líderes y animadores juveniles supone un momento destacado de la oferta formativa que complementa al trayecto escolar, experiencia presente en numerosos colegios de gestión privada.

\section{LA RECREACIÓN EDUCATIVA EN URUGUAY}

La recreación, entendida como intervención organizada, refiere a un movimiento que se desarrolla desde Norteamérica, con fuerte influencia en la mayoría de los países latinoamericanos, en el marco del proceso modernizador que se da en el continente a comienzos del siglo xx. En este periodo, se difundió un enfoque recreacionista en la mayoría de los países del continente, estrechamente ligado a la educación física y como prácticas higiénicas en el uso del tiempo libre. Sin embargo, este modelo no se ha mantenido inalterado.

Si bien podemos decir que el enfoque original de la recreación aún tiene vigencia en ciertas prácticas, encontramos en el campo recreativo de diversos países del continente cada vez más experiencias que dan cuenta de un cambio en la forma de intervenir desde la recreación. En este contexto de revisionismo, algunos autores de la región han acuñado el concepto de recreación educativa (Waichman, 2004; Lema, 1999; Osorio, 2001; Mesa, 1998), que engloba un conjunto de prácticas que buscan diferenciarse del enfoque recreacionista de los países anglosajones, que atienden a sus a componentes pedagógicos y didácticos de la intervención. Uruguay se encuentra entre estos países que están buscando su propio modelo de recreación, apelando a fundamentos pedagógicos desde una mirada más integral.

Actualmente, la recreación organizada en Uruguay refiere a un conjunto de prácticas diversas, que se dan en una gran variedad de ámbitos e instituciones. Si bien en muchos espacios suele ser una práctica asociada a la educación física, lo que implica priorizar juegos motrices que permiten dar a la población diversas opciones de disfrute, cada vez son más las propuestas que se asocian con prácticas educativas que van más allá de lo motriz, y configuran un campo de acción e intervención propio.

Como sucede en otros países del continente, estas prácticas educativas tienen un fuerte desarrollo en el ámbito comunitario. Pero, en el caso uruguayo, la recreación también ha adquirido una fuerte presencia en las instituciones de educación formal: importantes recursos se han destinado para llevar adelante estas propuestas que apuntan a la formación integral de la comunidad esco$\operatorname{lar}^{2}$. La presencia de la recreación en estas instituciones ha crecido en las últimas dos décadas y se ha convertido en un rasgo distintivo de la recreación en Uruguay ${ }^{3}$, no solo por el impacto que tiene en la transformación de un campo académico y profesional, sino también por la cantidad de gente que estas actividades movilizan.

Estas propuestas de recreación institucionalizadas en muchos colegios y liceos, en su mayoría situados en la capital del país (Montevideo), comprenden a diversas propuestas lúdicas, artísticas y deportivas, que se desarrollan desde un énfasis recreativo, y que procuran contribuir a la formación integral del sujeto. Son propuestas que acompañan el ciclo escolar, durante todo el año académico y a lo largo de los diferentes niveles de escolarización, y se constituyen en un trayecto formativo complementario y fundamental para la intencionalidad educativa de estas instituciones.

2 Por enseñanza escolarizada entendemos aquí a todas las instituciones de educación formal, en sus diferentes niveles (inicial, primaria, secundaria, terciaria). Si bien en un sentido amplio engloba a diversas instituciones de educación no formal, nos interesa concentrar nuestro análisis en las primeras, ya que entendemos que es desde estas instituciones que el campo recreativo se está definiendo como una práctica educativa.

3 Si bien no existen registros que den cuenta de la totalidad de instituciones escolares que cuentan con una propuesta de recreación, a través de un relevamiento de las páginas web de los principales colegios de Montevideo, pudimos identificar al menos 40 instituciones que cuentan con un espacio regular de formación de líderes y animadores. 
La presencia de la recreación en estas instituciones se potencia a través de algunas actividades claves, como son los campamentos y la formación de animadores juveniles. Los campamentos se encuentran a lo largo del ciclo escolar, como momentos intensificadores de la experiencia formativa; en tanto que la formación de animadores juveniles, suele ocupar la última etapa de escolarización, con una orientación al servicio interno pero con un impacto que trasciende a la institución.

Estas prácticas son reconocidas institucionalmente por el impacto significativo que logran en la formación de los sujetos que asisten a estas instituciones, y son valoradas como componente esencial de una formación integral. El desarrollo de procesos de formación desde una oferta educativa opcional permite estimular el desarrollo de competencias para la construcción de autonomía de niños y jóvenes.

El presente artículo es un avance de la investigación sobre la recreación educativa como trayecto de formación, que pretende analizar las prácticas de recreación organizada en las instituciones escolares del Uruguay.

\section{MARCO REFERENCIAL}

Hoy en día nos encontramos que uno de los principales ámbitos para el desarrollo de la recreación en Uruguay se da en las instituciones de educación formal, a través de una oferta variada de actividades de tiempo libre escolar que incluye campamentos, jornadas recreativas, propuestas deportivas y culturales, incluso actividades de animación pastoral en el caso de colegios católicos. El contexto particular en el cual se dan estas prácticas -instituciones de educación formal, que refieren al tiempo de obligaciones de la población infantil y juvenil-demanda una serie de precisiones desde lo conceptual.

En primer lugar, debemos encuadrar estas experiencias en el marco de prácticas organizadas de recreación y desde un enfoque educativo. La recreación educativa es entendida como una práctica pedagógica en y para el tiempo libre, o sea que el tiempo libre es ámbito y fin de la acción educativa. En esta línea, Waichman (2004) plantea que el verdadero potencial de la recreación está en considerarla como una práctica pedagógica, lo que supone una intervención institucionalizada: una acción organizada, estructuras que la sostengan, objetivos precisos y métodos específicos, docentes especializados, etc. Esto supone un currículum de formación, una acción organizada en un tiempo específico, con una metodología participativa y grupal.

En el entendido de que la formación es una acción del sujeto sobre sí mismo, pero que se ve potenciada por diversas mediaciones con el objeto de aprendizaje (Ferry, 1997), la recreación es una oportunidad para contribuir al desarrollo humano desde la autonomía. La mejor mediación que puede proporcionar un recreador supone crear las condiciones adecuadas para que se desarrollen las potencialidades del sujeto. Por ello, es importante concebir a la recreación como acción didáctica.

Hablar de intencionalidad educativa supone revisar las prácticas de la recreación organizada, buscar nuevas formas de organización de la actividad, ensayar nuevos métodos y redefinir el rol del recreador, desde parámetros que no sean los tradicionalmente impuestos por la educación física.

A diferencia de la tendencia recreacionista que impone la visión anglosajona, el enfoque educativo pone el foco en la vivencia de la recreación como facilitadora de una experiencia de formación del sujeto. En este sentido, la recreación educativa podríamos definirla como un modelo vivencial de educación del ocio (Cuenca, 2004) ya que es una intervención educativa a largo plazo, que se basa en situaciones, vivencias y experiencias formativas relacionadas con el tiempo libre. Se tratan de experiencias colectivas, que toman al sujeto en un contexto de grupalidad (compañeros de estudios, colegas de un club, miembros de una comunidad) y que involucra los intereses grupales desde una participación activa; experiencias que no solo buscan entretener, sino también atender integralmente al resto de las necesidades que se ponen en juego a través de una intervención recreativa, y que generan beneficios duraderos.

La perspectiva educativa de la recreación supone considerar las potencialidades del sujeto para alcanzar su máximo desarrollo. En este sentido, Osorio (2001) considera que la recreación es un satisfacer sinérgico que atiende integralmente las necesidades del sujeto, por lo que en su dimensión organizada se debe procurar la "creación de escenarios para el desarrollo humano" (Osorio, 2008) y dinamizar así procesos que faciliten una vivencia que impacte integralmente en todas las 
dimensiones del ser. La intervención recreativa debe promover la toma de conciencia de las verdaderas necesidades para el desarrollo humano de una comunidad, identificar los factores que limitan el desarrollo y construir satisfactores apropiados para esa situación y en una interacción positiva con el entorno. El punto de partida es, para Osorio (2008), lograr un estado de bienestar físico, mental o social que se alcanza a través de experiencias de ocio satisfactorias, por lo cual la recreación, en cuanto actividad placentera, genera las condiciones para iniciar ese proceso de desarrollo. Desde esa base, la recreación debe facilitar también el desarrollo de las capacidades humanas para la construcción de satisfactores.

En tanto el enfoque recreacionista toma al juego como un recurso para el disfrute, la recreación educativa se propone ir más allá movilizando la capacidad lúdica del sujeto para interactuar creativamente con el entorno. La recreación educativa se entiende como una acción que apunta a movilizar la capacidad lúdica del sujeto, lo que permite aspirar a prácticas pedagógicas transformadoras. En este sentido, el concepto de lúdica enriquece a la recreación y le permite trascender del rol instrumental del juego — técnicas lúdicas-, para avanzar hacia un modelo que privilegie el potencial transformador de esa capacidad lúdica. "La lúdica no como un medio, sino como un fin, debe ser incorporada a lo recreativo más como un estado ligado en forma natural a la finalidad del desarrollo humano, que como actividad ligada sólo al juego [...]" (Carreño, Rodríguez y Uribe, 2014, p. 59).

La recreación educativa, en cuanto práctica pedagógica, interviene tanto en las prácticas sociales de tiempo libre como en la actividad interna del sujeto, y moviliza de ese modo su capacidad lúdica y expresiva. Así lo entiende Mesa (2004), para quien la recreación plantea algunos mecanismos particulares de influencia educativa, ayuda pedagógica que el recreador brinda al sujeto para la construcción de sus propios aprendizajes y significados, en la interacción entre el recreador, los recreados y los contenidos de naturaleza lúdico-creativa (Mesa, 1998).

Desde esta forma de concebir a la recreación como práctica pedagógica transformadora, es que entendemos a la recreación educativa como un trayecto de formación que se puede dar en el tiempo libre escolar y a través de propuestas no formales de las instituciones escolares. Afirmar esto demanda problematizar los conceptos de tiempo libre y de educación no formal.
Si bien coincidimos con varios autores que demandan un abordaje cualitativo al concepto de tiempo libre (cf. Munné, 1992), a los efectos de este trabajo optamos por una definición operativa que considerará la dimensión cuantitativa de tiempo disponible para el sujeto. Desde esta perspectiva, el tiempo libre es definido como aquel que queda liberado del trabajo y otras obligaciones, y disponible para lo que el sujeto decida, es la porción del tiempo social, que queda luego de cumplir con el trabajo y otras obligaciones, abierta a cualquier ocupación que decida el sujeto (Trilla, 1993).

En el caso de la población beneficiaria de las propuestas de este estudio (jóvenes escolarizados entre 15 y 18 años), el tiempo de obligaciones suele ser el destinado a la actividad escolar, por lo que el tiempo libre sería aquel que queda disponible luego de la actividad académica y en el cual el sujeto elige según su voluntad y por el placer que la actividad le genera.

Atendiendo a esta definición, creemos que una variable que nos permite delimitar a la oferta recreativa en las instituciones es la del tiempo libre escolar, entendido como el tiempo que se destina dentro de la institución para actividades que no son académicas y que requieren la voluntad de participación por parte del sujeto. En Uruguay, la enseñanza escolar es obligatoria, por lo que el tiempo destinado a las actividades académicas corresponde a la esfera de las obligaciones. Sin embargo, hay una dimensión de tiempo institucionalizado, cuya dedicación no es obligatoria y que suele quedar a decisión del alumno la participación en ese espacio.

Este es un tiempo institucionalizado, ya que responde a una intencionalidad educativa y una estrategia de intervención en el tiempo disponible. A diferencia del tiempo académico, no es obligatorio, sino que implica una participación voluntaria. En este sentido, si bien se plantean objetivos educativos, la decisión de la participación obedece a una motivación intrínseca que no persigue otro fin que el disfrute de la actividad en sí misma. La motivación personal, la voluntad y el disfrute en el tiempo libre son características que definen a las actividades recreativas y de ocio (Cutrera, 1997; Trilla, 1993); en este sentido, lo que define a la oferta recreativa de estas instituciones es que se trata de actividades de tiempo libre institucionalizado, que se desarrollan generalmente fuera del horario obligatorio y en espacios más allá del aula, pero que son centrales para la orientación educativa de la institución. 
La oferta recreativa en la que se enmarca la formación de animadores se da, salvo excepciones, fuera del horario escolar o a contra-turno, es decir, en un horario opuesto al escolar e incluso los fines de semana. Ocupan parte del tiempo disponible de los participantes y ellos optan por ocupar su tiempo libre con estas actividades dentro de la institución escolar, sin que esto incida en su certificación escolar.

Estamos hablando de actividades diversas - como la práctica recreativa de algún deporte, talleres de expresión artística, campamentos, jornadas festivas, servicio voluntario, etc.-, en las que el énfasis es esencialmente recreativo, ya que el foco está en la experiencia que se da durante el proceso y en menor medida en el resultado.

Por otra parte, si bien estas propuestas se desarrollan en instituciones de educación formal, consideramos que la oferta recreativa se define desde la no formalidad, ya que engloba en buena medida a las actividades educativas que la institución realiza en forma intencionada, pero que no forma parte de la enseñanza oficializada, graduada y jerarquizada que, según Trilla ${ }^{4}$ (1993), caracteriza a la educación formal. Si bien estamos hablando de instituciones de educación formal, la institucionalidad no es el único parámetro para definir la formalidad de una propuesta. Grandstaff (citado por Waichman, 2004) plantea que la no formalidad puede darse desde la adscripción administrativa (institucionalidad), pero también desde el enfoque pedagógico, desde la función educativa, desde los beneficiarios a los que se dirige o desde la pertinencia cultural. En este caso, si bien la institucionalidad es formal, podemos decir que la no formalidad se da en los aspectos pedagógicos y metodológicos, en su funcionalidad y en la adaptabilidad cultural. La oferta recreativa introduce las virtudes de la educación no formal, en un contexto institucional escolarizado, en cuanto la flexibilidad en estilos pedagógicos, el desarrollo de métodos más específicos y adaptables, y el protagonismo de los participantes.

A diferencia de la actividad escolar, no son actividades que condicionen la aprobación del curso escolar y el pasaje de grado. En este sentido, la no formalidad de estas propuestas implica una disminución de los

4 Para este autor, el subsistema de educación no formal está "formado por el conjunto de instituciones y medios educativos intencionales y con objetivos definidos que no forman parte del sistema de enseñanza graduado o formal" (Trilla, 1993, p. 15). requerimientos externos, en cuanto a programas, burocracia y expectativas sociales. Suelen ser actividades no obligatorias, que no requieren aprobación para continuar progresando en el trayecto, pero que forman parte de la oferta curricular -en un sentido amplio ${ }^{5}$ - propuesta por la institución como estrategia de formación. Es decir que, si bien se organizan respondiendo a una lógica de no formalidad, creemos que tampoco es correcto denominarlas - como suele hacerse- actividades extracurriculares, ya que responden explícitamente a una intencionalidad educativa, y desarrolla una estrategia acorde que busca complementar y enriquecer la formación del sujeto.

El desarrollo y crecimiento de la oferta complementaria ha llevado en numerosas ocasiones a la necesidad de crear, generalmente en las generaciones mayores, un espacio de capacitación de recursos voluntarios para colaborar en las propuestas. Estas experiencias de formación de líderes y animadores, y su aporte a los trayectos de formación en instituciones educativas, es lo que pretendemos analizar en este artículo.

\section{ENCUADRE METODOLÓGICO DEL ESTUDIO}

La investigación se propuso desarrollar teoría a partir de la singularidad de las prácticas de recreación educativa que se dan en Uruguay. Para ello, nos concentramos en las representaciones de quienes gestionan esos programas, a partir de las formas en que construyen los discursos sobre sus prácticas - sus propias teorizaciones-, desde una metodología cualitativa.

5 Según Salinas (1998), podemos encontrar cuatro significados del concepto currículum. El más utilizado es el del currículum como documento, es decir, asociado a un cierto plan de estudios (pero que implica también tres dimensiones - aula, centro, políticas-). Una segunda acepción refiere al currículum como norma y supone un conjunto de pautas que organizan la actividad institucional y la actividad docente. Un tercer significado hace al currículum como experiencia y refiere a todo lo que se enseña explícita e implícitamente, lo que lo constituye en un proyecto que da sentido y coherencia a una oferta educativa. Un último significado del concepto sería el de currículum como proyecto cultural, lo que supone un cruce de prácticas diversas que definen los procesos de enseñanza y de aprendizaje. Esto implica la selección de criterios para asumir una filosofía educativa, objetivos, contenidos, aspectos formales, una evaluación, ciertas formas de relacionarse con la familia y la comunidad, de organización institucional, etc. 
En cuanto investigación cualitativa que busca desarrollar teoría a partir de un conjunto de prácticas singulares, hemos optado por situarnos en el enfoque de la grounded theory o teoría fundamentada. Este es un enfoque que nos permite crear propuestas teóricas basándonos principalmente en los datos que surge de la realidad estudiada, "una metodología general para desarrollar teoría que está enraizada (grounded) en información sistemáticamente recogida y analizada" (Strauss y Corbin, 1994 citado por Soneira, 2007, p. 153). Creemos que este es un enfoque adecuado para nuestra investigación ya que escogimos un objeto de estudio que no cuenta hoy con una teoría propia y, por lo tanto, esta debe surgir de las propias prácticas de los recreadores. "La teoría fundamentada es especialmente útil cuando las teorías disponibles no explican el fenómeno o planteamiento del problema [...]" (Creswell, 2009 citado por Hernández, Fernández y Baptista 2010, p. 492). De acuerdo con la metodología elegida, se procedió a desarrollar un proceso cíclico de recolección, codificación, análisis e interpretación de los datos.

Para la recolección de datos se optó por realizar entrevistas en profundidad a gestores y expertos de instituciones referentes de la recreación en Uruguay. Con estas entrevistas, buscamos relevar las representaciones que estos profesionales realizan de su propia práctica y su valoración de los aspectos pedagógicos y didácticos de la intervención. El producto de este avance se centra en diez entrevistas a referentes de instituciones que cuentan con formación de líderes y animadores juveniles, como un aspecto central de su propuesta recreativa, instituciones localizadas en el área metropolitana (que incluye a la ciudad de Montevideo y otras ciudades satélites en un radio de $30 \mathrm{~km}$ ).

Los entrevistados fueron seleccionados en primer lugar porque la institución en la que trabajan puede identificarse como caso representativo de la recreación educativa en el Uruguay. Pero, en tanto muchos de ellos han sido protagonistas del desarrollo de este enfoque, también nos interesó indagar en su perspectiva como expertos del campo estudiado. Las instituciones e informantes se fueron seleccionando por ser ejemplos representativos de buenas prácticas, por su historia destacada en el campo, por el desarrollo alcanzado de sus programas recreativos y atendiendo a la diversidad de perfiles institucionales antes mencionada.

\section{LA RECREACIÓN COMO TRAYECTO DE FORMACIÓN}

La recreación en instituciones escolares está sostenida por una oferta de actividades diversas y que abarca todo el ciclo escolar, desde el nivel inicial y la educación primaria hasta el ciclo básico y superior de la Educación Secundaria, comprendiendo un periodo de 15 años clave para la formación del sujeto (entre los 3 y los 18 años de edad). Esto implica una diversidad de propuestas que apuntan a atender facetas del trayecto formativo que no son suficientemente abordadas desde el aula tradicional. Podemos considerar, entonces, a la recreación en instituciones educativas como una estrategia que permite desarrollar un trayecto de formación complementario al escolar.

Por trayecto formativo entendemos el diseño de un proyecto de formación que posibilita recorridos a través de una progresión no siempre lineal que se da durante el ciclo escolar o una parte de este, y que comprende no solo a las actividades académicas. Hablar de trayectos de formación supone considerar algo más que los trayectos escolares ${ }^{6}{ }^{6}$ integrando también esta dimensión de actividades recreativas que se dan en la escuela pero por fuera del horario académico.

Este es un aspecto que enriquece el potencial educativo de estas experiencias y permite ir más allá del aprendizaje instrumental. La oferta recreativa no apunta a la transmisión de contenidos, sino a generar desde la voluntad del sujeto experiencias movilizadoras que tengan un impacto significativo en su desarrollo. Mediante estas propuestas recreativas, se sostiene una oferta a lo largo de todo el año y a lo largo del ciclo escolar, lo que permite al niño y al adolescente trazar su trayecto formativo específico con base en las elecciones que va tomando durante su proceso de desarrollo. Cuanto mayor es la oferta de actividades, más personal se vuelve su trayectoria.

6 La trayectoria escolar alude al diseño de itinerarios que refieren a la enseñanza escolarizada. Un conjunto complejo de factores incide en "[...] las múltiples formas de atravesar la experiencia escolar, muchas de las cuales no implican recorridos lineales por el sistema educativo" (Maddonni, 2009, p. 19). En la medida que el trayecto escolar suele asociarse a las actividades académicas, en nuestro caso optamos por hablar de trayecto formativo lo que englobaría al trayecto escolar y al trayecto formativo complementario que es sostenido desde la oferta recreativa, complementando la experiencia educativa dentro de la institución y con impacto significativo en el desarrollo integral del sujeto. 
El presente artículo se centra en uno de los aspectos más destacados de los trayectos formativos relevados, que es la formación de líderes y animadores juveniles, experiencia que se da en los últimos años escolares y que permite potenciar las trayectorias personales al tiempo que da sostenibilidad a toda la propuesta.

\section{LA FORMACIÓN DE ANIMADORES}

La formación de animadores ${ }^{7}$ es una instancia sistemática que se inicia generalmente en el tercer o cuarto año de Secundaria y que se mantiene durante los años siguientes, con el objetivo de formar un voluntariado hacia la labor interna en la propia institución. Desde la escuela de animadores se promueven no solo actividades de formación, sino también eventos vivenciales y de complemento a la formación, además de tareas de extensión dentro de la institución.

La organización de la escuela en dos niveles permite diferenciar una propuesta inicial, durante el primer año, centrada en la conformación grupal y en la adquisición de recursos para el desempeño como animador de actividades recreativas. Este nivel se complementa, durante los años siguientes, con un grupo orientado al servicio y la extensión dentro y fuera de la institución, donde se da una mayor reflexión y compromiso con las

7 La denominación varía en algunos casos se habla de líderes, incluso en otros de recreadores. Esto está generalmente vinculado, según surge del relevamiento, por diversas tradiciones que influyen en cada institución. En el caso de las instituciones que utilizan la denominación liderato, es explícita la influencia de educadores formados por la Asociación Cristiana de Jóvenes (YMCA), de raíz anglosajona. El concepto de animación es utilizado en la mayoría de las instituciones, muchos de ellos colegios católicos vinculados a educadores formados en el movimiento scout, que en el Uruguay han sido fuertemente influenciados por la tradición francesa. El concepto de recreador es utilizado en menor medida, en algunos casos por influencia de la educación física y con un enfoque recreacionista. En nuestro caso, consideramos que cada denominación alude a una etapa diferente en la trayectoria del sujeto: liderazgo sería una cualidad que algunos de los niños y adolescentes desarrollan durante su trayecto formativo, lo que los llevaría en su última etapa escolar a asumir un rol de animador de carácter voluntario. Luego de culminada la etapa escolar, algunos de estos animadores suelen seguir su vínculo con la institución, esta vez en forma remunerada, lo que implicaría asumir un rol de recreador profesional. Este artículo se centra en esa etapa intermedia de formación y desempeño como animadores, por lo que optamos por esta denominación para englobar a todos los casos más allá de la denominación por la que optan. actividades institucionales. Este segundo nivel puede concluir al finalizar el ciclo escolar, o incluso proyectarse más allá del egreso.

Gráfico 1. Escuela de animadores

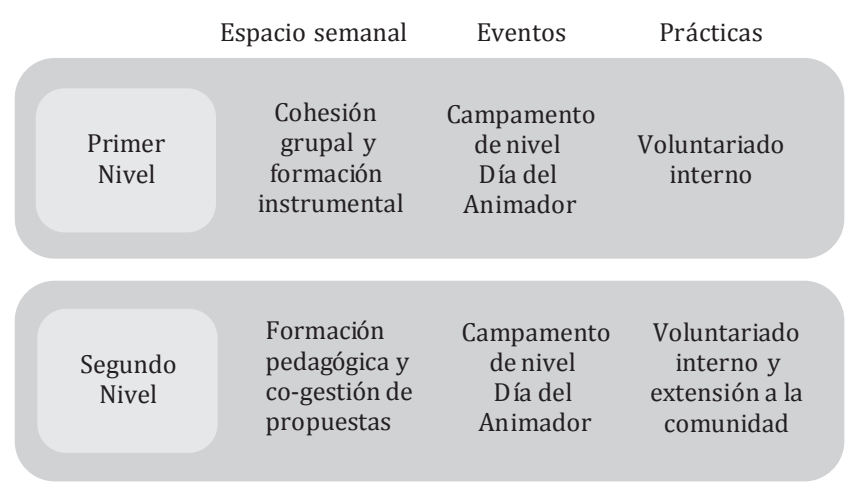

Fuente: elaboración propia.

Lo que llamamos la parte curricular o de talleres es una vez por semana, una hora y media sistemáticamente. [...] Y después otra pata es lo que llamamos actividades especiales de formación, entonces bueno hay uno, dos campamentos de animación, [...] una jornada sobre el juego que hacemos con ellos, el día del animador, una ceremonia de clausura que a su vez tiene todo unos condimentos y es un (momento) muy fuerte para la vida de los animadores. Y la tercera lo llamamos momento de prácticas recreativas donde ellos acompañan en distintas instancias, en algunas puntuales y en otras más sistemáticas: 4to en general acompaña a Primaria al deporte y también a clases de educación física de los más chicos, hasta 2do año de escuela; el 2do nivel acompaña más a lo que es Ciclo Básico, también en algunos casos acompaña a deporte, pero más que nada campamentos, jornadas especiales, actividades de pastoral. (E10).

Pese a ser una propuesta que implica una dedicación importante, la participación en esta instancia suele ser alta, en algunas instituciones alcanza el primer nivel con un $50 \%$ de los estudiantes de su generación. Esto es coherente con el éxito de la oferta recreativa previa, que lógicamente favorece la participación en esta propuesta, ya que los niños y adolescentes recorren todo el itinerario escolar en contacto con animadores y llegan a bachillerato con la motivación de acceder a ese rol.

Esta formación de animadores cumple con una triple función: formar un cuerpo de voluntariado interno, destinado a sostener el desarrollo de toda la oferta recreativa en los diferentes tramos del ciclo escolar; 
facilitar la inserción laboral temprana de los jóvenes a punto de egresar, desarrollando recursos y habilidades para sus primeras experiencias de trabajo; y sobre todo contribuir a profundizar y proyectar la formación integral del adolescentes, consolidando las competencias desarrolladas a lo largo del recorrido por la institución educativa.

Contar con un importante número de animadores facilita la posibilidad de desarrollar una propuesta recreativa variada y con una cobertura importante del ciclo escolar. Los animadores son un soporte fundamental para las actividades recreativas de todos los niveles en la institución educativa. Desde la educación inicial, pasando por la primaria e incluyendo la secundaria, los animadores colaboran en los diferentes espacios y eventos de recreación (jornadas, talleres, campamentos, convivencias, fiestas, etc.), haciendo posible una amplia y diversa cobertura para la oferta recreativa institucional.

Es una actividad, como decimos nosotros, de voluntariado interno al colegio, el devolverle a la institución lo que ellos (los animadores) pudieron recibir, gracias a que hubo otros chicos que estuvieron; ahora a ellos les permiten acompañar a los otros. (E1).

La formación de animadores hace posible contar con recursos humanos voluntarios, sin los cuales sería difícil sostener una oferta tan diversa. Por ello, entendemos a esta propuesta como una actividad meta-recreativa, ya que no solo se trata de una actividad recreativa en sí misma, sino que además hace posible al conjunto de la oferta de formación complementaria. Es una instancia de meta-recreación, ya que permite a la oferta recreativa reproducirse a sí misma y sostener las trayectorias educativas que a través de esta se desarrollan.

\section{LA ANIMACIÓN COMO ACTIVIDAD DE FORMACIÓN DEL SUJETO}

Esta función de voluntariado interno es fundamental, pero no es la principal. Lo que le da sentido a la propuesta es la función que cumplen en la formación personal del propio adolescente, como culminación de un trayecto de formación integral a lo largo del ciclo escolar.

Más allá de la formación específica como animador, creemos que estos espacios de formación en estas instituciones educativas se constituyen en propuestas calificadas dentro de la oferta de formación integral. Es una propuesta calificada pues implica una carga horaria importante, una mayor proximidad en el vínculo con los educadores, especialmente porque apunta a la apropiación por parte del sujeto de su propio proceso de formación.

\begin{abstract}
Me parece que el animador cuando se sienta en una mesa a programar junto con el profe, pasa a ser no ya el alumno, pasa a hablar con el profe de igual a igual, dentro de lo que puede ser y lo acerca a la institución de tal manera de ayudar a algo, por eso me parece que es tan importante eso, que es la famosa convivencia. (E6).
\end{abstract}

La participación en la escuela de animadores implica destinar una importante cantidad de tiempo, por fuera del horario escolar, pues supone una instancia de encuentro semanal de formación y preparación de actividades, la participación en eventos específicos (como el Día del Animador o Campamento de Animadores) y la realización de prácticas voluntarias dentro de la propia institución (asistiendo la actividad deportiva, colaborando en la realización de jornadas recreativas y animando los campamentos de las generaciones más chicas). Estos jóvenes dedican voluntariamente buena parte de su tiempo disponible, para las instancias de formación, y para la preparación y ejecución de las diversas actividades en las que les toca participar, con lo que convierten su tiempo libre en una oportunidad para desarrollar experiencias formativas desde la gratuidad.

En estos espacios, además, logran desarrollar un vínculo más cercano con los recreadores y otros educadores, compartiendo tareas y cogestionando las propuestas. Los jóvenes aprenden a establecer relaciones laborales con adultos, desde la corresponsabilidad, y se acercan así a las exigencias de la vida adulta.

Y cuando los gurises ingresan al cuerpo de líderes, ingresan al grupo, al núcleo más duro del Colegio [...], al grupo de gente que vos sabes que podes contar, yo con ellos y con los cuales en ellos se transmite esta idea de Colegio y esta concepción de ser persona y ser institución... (E8).

La alta dedicación dentro de la institución, que va más allá del horario escolar, supone que estos jóvenes ocupen los tiempos y espacios de la institución, que se apropien de esos espacios, y los resignifiquen. El empoderamiento 
que se logra permite la construcción de un ambiente educativo y una experiencia de formación muy calificada, que permite llevar el trayecto formativo hacia sus mejores posibilidades.

Pero, especialmente, la participación como animadores permite una oportunidad privilegiada de profundizar en su formación y desarrollo personal, y proyecta este más allá del ciclo escolar. $\mathrm{Al}$ asumir un rol de animador, el espacio de capacitación pasa a un segundo plano. Lo relevante no es formar voluntarios, sino la experiencia formativa que vive el sujeto en sí mismo, como última etapa de su trayecto formativo personal e integral.

\section{EL ANIMADOR COMO MEDIACIÓN PEDAGÓGICA}

Otro aspecto a destacar es el aporte pedagógico de este rol, a la función educativa de estas instituciones. El animador juvenil se constituye en un actor clave en estos colegios, no solo por sostener una oferta tan diversa, sino también porque cumple con un rol pedagógico clave para el proceso formativo del resto de los alumnos. A partir de la participación en las propuestas recreativas de las generaciones más jóvenes, el animador establece un vínculo educativo con sus pares más pequeños, donde la proximidad etaria permite establecer vínculos más estrechos, en función de intereses compartidos. La propuesta de formación de animadores no solo potencia el trayecto formativo del animador, sino que también potencia las trayectorias formativas de los alumnos más jóvenes, construye de este modo un rol de educador cercano al alumno y con una mirada particular hacia ese espacio complementario de formación.

A través del rol del animador, los niños y adolescentes entran en contacto con sus mayores, que se convierten en un modelo de referencia para sus propias trayectorias. Estas trayectorias se proyectan en la figura del animador juvenil. La propia trayectoria del animador se vuelve proyecto de formación en el cual los menores pueden reflejarse. De esta forma, el animador es un actor que cuenta con un gran reconocimiento, no solo de los alumnos, sino también de los demás educadores adultos de la institución.

Existe consenso en considerar al animador como un educador más de la institución, que facilita la mediación de los procesos educativos y hace de puente entre los adultos y niños de la institución. De forma implícita, al animador le compete la función de trasponer didácticamente los objetivos formativos de la institución. ${ }^{8}$ En este sentido, el animador contribuye a transformar los objetivos de formación en competencias desarrolladas, a través de su labor de mediación y gracias a una mayor cercanía tanto con los niños como con los educadores.

Ahora, es interesante porque (los animadores) son los que deben hacer la síntesis entre lo que las maestras quieren y los gurises quieren. Ahí hay que hacer ese proceso de transformar objetivos en actividades y transformar intereses educativos en intereses del propio grupo. (E9).

Articulador entre la niñez y la adultez. (E6).

Acorde con una mirada progresiva, algunas instituciones han apostado por este rol para dinamizar el trayecto formativo, asignándole la tutoría de alguno de los grados. En este caso, el animador asume una suerte de tutoría del grupo, y participa de toda la oferta recreativa que se desarrolla a lo largo del año con el mismo. Este rol voluntario de tutor de grupo tiene un impacto favorable, tanto en el proyecto de formación de cada nivel como en la trayectoria formativa del propio animador. Contribuye a potenciar sinérgicamente sus necesidades de participación, afecto, entendimiento, identidad, creación, en especial de autonomía, y es una instancia muy significativa en la culminación de su experiencia formativa dentro de la institución.

Por otra parte, además de voluntariado interno, la formación de animadores es una oportunidad de desarrollar extensión a la comunidad. A partir de este espacio de formación, se suelen constituir grupos asociativos y de voluntariado, que desarrollan una veta social y solidaria, lo que le da un plus a la trayectoria formativa y una continuidad que se proyecta más allá del egreso del ciclo escolar.

\section{CUANDO LAS RAIICES SE VUELVEN ALAS (CONCLUSIONES)}

La formación de animadores es la culminación del trayecto de formación del sujeto a lo largo de su pasaje por la institución educativa. En este trayecto progresivo

8 La transposición didáctica refiere a las mediaciones que hacen los diferentes actores de la educación que se sitúan entre los objetivos educativos y lo que efectivamente se aprende (Frigerio, 1991) 
hacia la construcción de autonomía, la propuesta de animadores juveniles que se sitúa al final del ciclo escolar se constituye en el momento cumbre del proceso formativo, donde las raíces que se desarrollaron en la institución se vuelven alas. ${ }^{9}$

Esta propuesta se convierte en un factor clave para el éxito de la oferta recreativa en instituciones escolares y en un aspecto que potencia las posibilidades de formación. Esta macropropuesta tiende a ser un espacio privilegiado que potencia la experiencia formativa, le permite al sujeto recorrer la última etapa del trayecto y lo acerca a la anhelada construcción de autonomía.

El reconocimiento que cuentan los animadores juveniles en estas instituciones educativas y el espacio que se les otorga, llevan a considerar a estos jóvenes como una élite dentro de la institución, en cuanto a las posibilidades que se les brinda y las expectativas que generan. La importancia de este espacio para las instituciones y lo que a partir del mismo se genera, hacen de la formación de animadores una propuesta distintiva de la oferta recreativa y en un factor de identificación institucional.

El espacio de animadores juveniles y la propuesta recreativa que a partir de ellos se genera aporta de manera significativa a la configuración de un modelo singular de recreación educativa que hasta ahora no había sido suficientemente abordado por la investigación educativa.

El presente estudio permitió conocer una realidad particular de la recreación educativa y brinda algunas pistas para comprender el potencial del desarrollo de proyectos de formación a través de la oferta recreativa, que enriquezca las oportunidades educativas en instituciones escolares. Pese a ser un estudio acotado a la ciudad de Montevideo y las instituciones de gestión privada, consideramos que da cuenta de una realidad bastante significativa en nuestro país y que puede aportar elementos para el desarrollo de experiencias similares en otros contextos.

9 Frase que se utiliza en una de las instituciones relevadas para representar el egreso.

\section{REFERENCIAS}

Carreño, J. M., Rodríguez, A. B., y Uribe, J. J. (2014). Recreación, ocio y formación. Armenia: Kinesis.

Cuenca, M. (2004). Pedagogía del ocio, modelos y propuestas. Bilbao: Universidad de Deusto.

Cutrera, J. C. (1997). Recreación: fundamentos, didáctica y recursos. Buenos Aires: Stadium.

Ferry, G. (1997). Pedagogía de la formación. Buenos. Aires: Novedades Educativas.

Frigerio, G. (1991). Currículum: norma, intersticios, transposición y textos. En G. Frigerio (comp.), Currículum presente, ciencia ausente: Normas, teorías y críticas. Buenos Aires: Miño y Dávila Editores.

Hernández, R., Fernández, C., y Baptista, P. (2010). Metodología de la investigación. México: Mc Graw Hill.

Lema, R. (1999). Recreación, tiempo libre y educación en el Uruguay. Revista Prisma, 11, 136-142.

Maddonni, P. (2009). Las trayectorias escolares: del problema individual al desafío de política educativa. Buenos Aires: Ministerio de Educación.

Mesa, G. (1998). La recreación como proceso educativo (conferencia inédita). Recuperada de www. redcreacion.org/documentos/congreso5/GMesa.htm

Mesa, G. (2004). La recreación "dirigida": ¿mediación semiótica y práctica pedagógica? (documento inédito), Cali: Universidad del Valle.

Munné, F. (1992). Psicosociología del tiempo libre. México: Trillas.

Osorio, E. (2001). Los beneficios de la recreación desde una perspectiva del desarrollo humano. Recuperado de www.redcreacion.org/simposio2vg/EOsorio.htm

Osorio, E. (2008).Educación para la recreación, una apuesta por la autonomía y la libertad para la transformación social. Recuperado de www.redcreacion.org/ documentos/congreso10/EOsorio.html

Salinas, D. (1998). Currículum, racionalidad y discurso didáctico. En M. Poggi (ed.), Apuntes y aportes parta la gestión curricular, (21-59). Buenos Aires: Kapelusz.

Soneira, A. J. (2007). La "Teoría fundamentada en los datos" (grounded theory) de Glaser y Strauss. En I. Vasilachis de Gialdino (Coord.), Estrategias de investigación cualitativa (pp. 153-174). Buenos Aires: Gedisa.

Trilla, J.(1993). Otras educaciones. Animación sociocultural, formación de adultos y ciudad educativa. Barcelona: Anthropos.

Waichman, P. (2004). Tiempo libre y educación, un desafío pedagógico. Buenos Aires: PW. 